Ann. Zootech., I974, 23 (2), I6I-169.

\title{
VALEUR ALIMENTAIRE DE LA FARINE DE BANANE ET DE MANIOC DANS LE RÉGIME DU PORCELET SEVRÉ A 5 SEMAINES : INFLUENCE DU TAUX DE PROTÉINES DE LA RATION
}

\author{
J. LE DIVIDICH et I. CANOPE
}

aveć la collaboration technique de $\mathrm{E}$. Despois et E. CALIF

Station expérimentale de Zootechnie, Centre de Recherches agronomiques des Antilles et de la Guyane, I. N.R. A., 97170 Petit-Bourg (Guadeloupe)

\section{RÉSUMÉ}

La farine de pulpe de banane verte incorporée à un taux voisin de 50 p. Ioo à la ration de sevrage du porcelet entre 5 et 9 semaines d'âge permet des performances de croissance comparables à celles obtenues avec la farine de manioc (racines entières séchées).

L'utilisation digestive apparente des principaux éléments (sauf les cendres) du régime à base de farine de banane est toutefois significativement inférieure $(\mathrm{P}<\mathrm{O}, \mathrm{OI})$ à celle du régime renfermant la farine de racines de manioc.

Les meilleures performances de croissance sont obtenues avec une ration présentant un taux de protéines voisin de 21,5 p. I0o. Un taux plus faible (19,0 p. IOo) ne permet ni une croissance maximale ni une bonne efficacité de la ration.

\section{INTRODUCTION}

L,e sevrage du porcelet consiste à lui supprimer, soit progressivement, soit brutalement, le lait de sa mère et à le remplacer par un aliment concentré presque exclusivement à base de produits amylacés. Le choix des constituants de l'aliment doit faire l'objet d'une attention particulière. Le jeune porcelet est en effet mal adapté à digérer 1'amidon en raison d'une sécrétion insuffisante d'amylase pancréatique (BAILEY, KitTs et Woods I956; WALKER I959). Ainsi, le jeune animal est sensible à l'état de dégradation de l'amidon (Cunningham, I959, Cunningham et BRIsson, I957) et il a des possibilités limitées de dégrader certains amidons tropicaux parti- 
culiers (AUmaitre, Corring et LE Dividich, I969). De plus, on sait que l'amidon non dégradé dans l'intestin grêle peut conduire à une diarrhée fermentaire dont les répercussions pathologiques sont graves chez le nourrisson (WEIJERS et KAMER, I965).

Dans de nombreux pays les rations de sevrage sont généralement constituées d'une proportion élevée de céréales (SMI'TH et LuCAS, I957 ; SaLmon-LEGaGneur et FEVRIER, I959) et dans certaines régions tropicales où la production céréalière est déficitaire ou inexistante, le manioc et les excédents de banane peuvent constituer des produits de remplacement. Néanmoins, si la valeur alimentaire du manioc est connue (Terroine, I93I; Oyenuga, i96I ; Aumaitre, i969), on ne possède par contre aucune donnée sur l'utilisation de la farine de banane séchée, par le porcelet sevré précocement. On sait seulement qu'elle est bien utilisée par le porc en croissance (SEVE, LE DIVIdich et CANOPE, I972).

C'est pourquoi nous avons comparé la valeur alimentaire de la farine de banane séchée, à celle du manioc, incorporée à des taux voisins de $50 \mathrm{p}$. Ioo dans les régimes et tenté d'étudier le taux optimum de protéines de telles rations.

\section{MA'TÉRIEL E'T MÉ'THODES}

\section{Animaux}

62 porcelets de race Large White, provenant de I 3 portées de notre élevage expérimental, sont utilisés dans 2 expériences successives décrites au tableau I.

TABLEAU I

Schéma expérimental et effectifs d'animaux

\begin{tabular}{|c|c|c|c|c|c|}
\hline Expérience & \multicolumn{2}{|c|}{ I } & \multicolumn{3}{|c|}{ II } \\
\hline Source d'amidon & Manioc & Banane & Manioc & & \\
\hline $\begin{array}{l}\text { P. } 100 \text { de protéines } \\
\text { dans la ration }\end{array}$ & 22 & 22 & 19 & 19 & 22 \\
\hline $\begin{array}{c}\text { Nombre de couples } \\
\text { d'animaux }\end{array}$ & 8 & 8 & 5 & 5 & 5 \\
\hline
\end{tabular}

Dans la première, 9 portées sont utilisées; au sein de chacune d'elles, 2 lots de 2 animaux chacun de même poids (I mâle et I femelle) sont constitués afin de comparer les régimes à base de farine de banane et de manioc.

Dans la seconde, 5 portées sont réparties suivant le même dispositif pour étudier l'effet de 2 taux de protéines (r9 et 22 p. гоo) sur les performances de croissance des animaux recevant les régimes à base de farine de banane ou de manioc.

Le lot recevant le régime à base de banane au taux de 22 p. Ioo de protéines constitue le lot de référence dans les deux expériences.

\section{Aliments}

La ration de sevrage contient, après mélange des constituants, environ 50 p. Ioo de farine (racines entières séchées) de manioc de Madagascar ou de farine de pulpe de banane verte déshydratée. Les aliments sont granulés à $2,5 \mathrm{~mm}$ de diamètre et distribués à volonté dans de petits 
nourrisseurs automatiques. La digestibilité apparente de quelques éléments de la ration (matière sèche, matière organique, azote) est estimée par une addition de o,3 p. Ioo d'oxyde de chrome, utilisé comme marqueur.

La composition chimique des produits de base et des aliments fabriqués est déterminée (tabl. 2 et 3 ). On peut souligner que la teneur en protéines brutes $(N \times 6,25)$ de la banane est environ deux fois plus élevée que celle du manioc. Les régimes contiennent entre 30 et 40 p. 100 d'amidon et leurs teneurs en protéines sont en moyenne de I9,0 et 2I,5 p. Ioo.

\section{TABLEAU 2}

Composition chimique des produits de base (en p. Iоo)

\begin{tabular}{|c|c|c|}
\hline & Farine de manioc & Farine de banane \\
\hline Matière sèche $\ldots \ldots \ldots \ldots \ldots$ & 86,6 & 87,0 \\
\hline Cendres $\ldots \ldots \ldots \ldots \ldots \ldots \ldots$ & 2,7 & 2,3 \\
\hline Protéines $(N \times 6,25) \ldots \ldots \ldots$ & 2,0 & 4,5 \\
\hline Cellulose (Weende) .......... & 2,2 & 1,2 \\
\hline 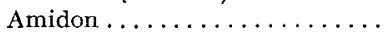 & 75,3 & 72,3 \\
\hline
\end{tabular}

TABLEAU 3

Composition et caractéristiques des aliments

\begin{tabular}{|c|c|c|c|c|}
\hline Régime (1) & 1 & 2 & 3 & 4 \\
\hline $\begin{array}{l}\text { Farine de banane } \ldots \ldots \ldots \ldots \\
\text { Farine de manioc } \ldots \ldots \ldots \ldots\end{array}$ & $\begin{array}{c}50,0 \\
\longrightarrow\end{array}$ & $\begin{array}{c}57,5 \\
\longrightarrow\end{array}$ & 50,0 & 54,8 \\
\hline $\begin{array}{l}\text { Sucre } \ldots \ldots \ldots \ldots \\
\text { Huile d'arachide } \ldots \ldots \ldots \ldots \\
\text { Complément minéral. } \ldots \ldots \ldots \\
\text { Complément vitamines }+ \text { anti- } \\
\quad \text { biotiques } \ldots \ldots \ldots \ldots \ldots \ldots \\
\text { Oxyde de chrome } \ldots \ldots \ldots \ldots \\
\text { Complément azoté }{ }^{(2)} \ldots \ldots \ldots\end{array}$ & $\begin{array}{r}1,5 \\
0,3 \\
37,7\end{array}$ & $\frac{1,5}{30,2}$ & $\begin{array}{r}1,5 \\
0,3 \\
37,7\end{array}$ & $\begin{array}{l}1,5 \\
- \\
33,2\end{array}$ \\
\hline Résultats d'analyses (p. 100) & & & & \\
\hline $\begin{array}{l}\text { Matière sèche } \ldots \ldots \ldots \ldots \ldots \\
\text { Cendres } \ldots \ldots \\
\text { Protéines }(\mathrm{N} \times 6,25) \ldots \ldots \\
\text { Amidon } \ldots \ldots \ldots\end{array}$ & $\begin{array}{r}87,6 \\
6,2 \\
21,8 \\
31,5\end{array}$ & $\begin{array}{r}87,5 \\
5,9 \\
19,2 \\
40,2\end{array}$ & $\begin{array}{r}88,1 \\
6,0 \\
21,2 \\
37,0\end{array}$ & $\begin{array}{r}88,6 \\
5,8 \\
18,8 \\
41,4\end{array}$ \\
\hline
\end{tabular}

(1) Expérience 1 : régimes 1 et 3 ;

Expérience 2 : régimes 1,2 et 4 (daus le régime 1 on remplace l'oxyde de chrome par 0,3 p. 100 de farine de banane).

(2) Composition p. 100 : Tourteau de soja à 44 p. 100 de protéines : 70 ; farine de poisson de Norvège :16; poudre de lait écrémé : 14. 


\section{Mesures}

Pour chaque lot expérimental, pendant $\mathrm{I}$ mois après le sevrage (du $35^{\mathrm{e}}$ au $63^{\mathrm{e}}$ jour) le gain de poids individuel des animaux et la quantité d'aliments consommée (pour 2 animaux) sont mesurés chaque semaine. L'efficacité alimentaire et le coefficient d'efficacité protidique sont calculés pour l'ensemble de la période.

L'état sanitaire des animaux a été noté et les résultats exprimés en jours de diarrhée déterminés de visu et rapportés par lot pour toute la durée de l'expérience. Pour la mesure de la digestibilité, un échantillon représentatif de fèces est prélevé chaque jour pour chaque lot et séché immédiatement à $70^{\circ} \mathrm{C}$. Les échantillons sont ensuite rassemblés pour une semaine d'expérience. L'azote total est dosé par la méthode Kjeldahl et l'oxyde de chrome par dosage colorimétrique (BoLIN et al., I952) après minéralisation en milieu sulfuro-perchlorique. L'amidon vrai est dosé par voie enzymatique à l'aide d'une glucoamylase (ThIvend et al., I965).

\section{RÉSULTATS}

Les performances du lot de référence (farine de banane à 22 p. Ioo de matières azotées) étant semblables dans les 2 essais : gain moyen quotidien 453 et $464 \mathrm{~g}$, efficacité alimentaire 0,50 et 0,53 , respectivement pour les essais I et 2 , les résultats des deux expériences sont regroupés et interprétés ensemble. L'interaction (source d'amidon $\times$ taux de protéines) est calculée selon SNEDECOR et CochraN (I97I) en tenant compte des différences d'effectif dans chaque traitement.

\section{I. - Vitesse de croissance et état sanitaire}

Les performances de croissance étant identiques (tabl. 4) pour le lot témoin, les performances sont comparées entre traitements et l'on peut calculer l'effet de la source d'amidon ainsi que du taux de protéines. Dans les conditions de notre expérience, on ne constate pas d'effet de la nature de la source d'amidon (farine de banane

TABLEAU 4

Vitesse de croissance des porcelets

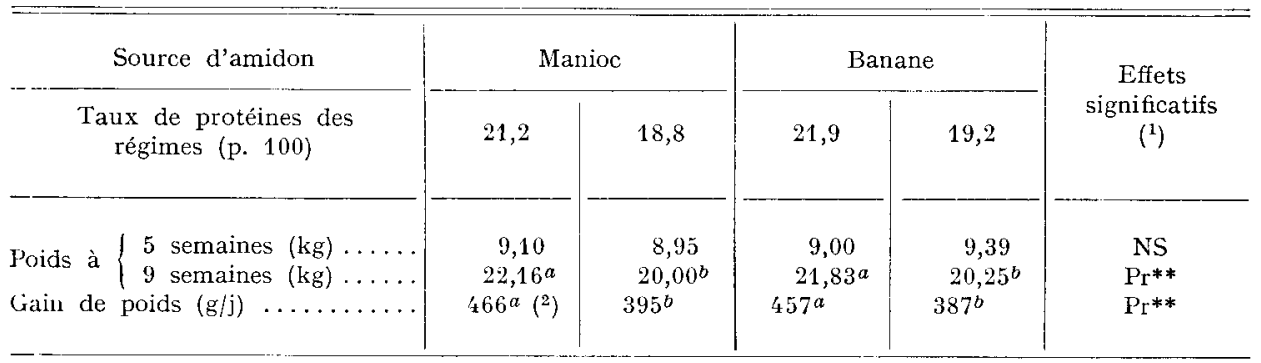

( ${ }^{1}$ Effets protéines : $\operatorname{Pr}$; effet source d'amidon : A; l'intéraction protéine $\times$ source d'amidon : $\operatorname{Pr} \times \mathrm{A}$; * : seuil de signification 0,$05 ; * *$ : seuil 0,$01 ;$ NS : non significatif.

$\left({ }^{2}\right)$ Les valeurs affectées de la même lettre ne sont pas significativement différentes entre elles au seuil 0,01 . 
ou manioc). On observe, par contre, un effet significatif du taux de protéines sur la croissance des animaux : 1'abaissement à 19 p. roo entraîne une diminution significative $\left(\mathrm{P}<\mathrm{O}\right.$,OI) d'environ $\mathrm{I}_{5} \mathrm{P}$. Ioo du gain de poids moyen journalier.

Par ailleurs, l'état sanitaire des animaux est très bon dans l'ensemble. Des diarrhées ne sont observées que pendant la semaine qui suit le sevrage; elles sont en outre très limitées en nombre, soit 2,7 et 0,9 jours respectivement pour les régimes à base de farine de manioc et de farine de banane.

\section{2. - Quantités d'aliments consommés. \\ Efficacité alimentaire et coefficient d'efficacité protidique}

Les divers résultats sont rapportés dans le tableau 5. Les quantités moyennes d'aliments consommés sont importantes, soit environ $900 \mathrm{~g} / \mathrm{j}$. Elles sont inférieures de $3 \mathrm{p}$. Ioo en moyenne pour les régimes à base de banane et de $5 \mathrm{p}$. Ioo pour le plus faible taux de protéines; cependant, les différences ne sont pas significatives.

TABLEAU 5

Quantités d'aliments consommés, efficacité alimentaire et protidique

\begin{tabular}{|c|c|c|c|c|c|}
\hline \multirow{2}{*}{$\begin{array}{l}\text { Source d'amidon } \\
\text { p. } 100 \text { de protéines }\end{array}$} & \multicolumn{2}{|c|}{ Manioc } & \multicolumn{2}{|c|}{ Banane } & \multirow{2}{*}{$\begin{array}{c}\text { Effets } \\
\text { significatifs } \\
\text { (1) }\end{array}$} \\
\hline & 21,2 & 18,8 & 21,9 & 19,2 & \\
\hline $\begin{array}{l}\text { Quantités d'alimønts } \\
\text { consommés par porcelet }\end{array}$ & & & & & \\
\hline $\begin{array}{l}\text { Totales }(k g) \ldots \ldots \ldots \ldots \ldots \ldots \ldots \\
\text { Journalières }(g) \ldots \ldots \ldots \ldots \ldots \ldots\end{array}$ & $\begin{array}{l}26,1 \\
934\end{array}$ & $\begin{array}{l}24,6 \\
878\end{array}$ & $\begin{array}{l}25,0 \\
890\end{array}$ & $\begin{array}{l}24,0 \\
857\end{array}$ & $\begin{array}{l}\text { NS } \\
\text { NS }\end{array}$ \\
\hline $\begin{array}{l}\text { Efficacité ailmentaire }\left({ }^{2}, \ldots \ldots \ldots\right. \\
\text { Efficacité protidique }\left({ }^{3}\right) \ldots \ldots \ldots\end{array}$ & $\begin{array}{l}0,50^{a} \\
2,35\end{array}$ & $\begin{array}{l}0,45^{b} \\
2,40\end{array}$ & $\begin{array}{l}0,51^{a} \\
2,33\end{array}$ & $\begin{array}{l}0,45^{b} \\
2,36\end{array}$ & $\begin{array}{l}\mathrm{Pr}^{*} \\
\mathrm{NS}\end{array}$ \\
\hline
\end{tabular}

(1) $\operatorname{Pr}^{*}$ : effet significatif du taux de protéines $\mathrm{P}<0,05$.

$\left.{ }^{2}\right)$ Efficacité alimentaire $=\frac{\text { gain de poids }(\mathrm{kg})}{\text { aliment consommé }(\mathrm{kg})}$.

$\left({ }^{3}\right)$ Coefficient d'efficacité protidique $=\frac{\text { gain de poids }}{(\mathrm{kg})}$

L'efficacité alimentaire des régimes à base de manioc et de banane est la même, elle augmente significativement $(\mathrm{P}<0,05)$ de Io $\mathrm{p}$. Ioo lorsque le taux de protéines s'accroît. En revanche, le coefficient d'efficacité protidique a tendance à diminuer légèrement lorsque le taux de protéines augmente, les différences ne sont toutefois pas significatives.

\section{3. - Digestibilité de quelques éléments des régimes (tab1. 6)}

On détermine un coefficient d'utilisation digestive apparente moyen sur toute la période expérimentale. La moyenne arithmétique des 4 valeurs successives est retenue pour comparer entre eux les deux régimes. 


\section{TABLEAU 6}

Coefficient de digestibilité apparente des différents éléments de la ration (exp. 1) (Valeurs moyennes non pondérées obtenues sur la période totale)

\begin{tabular}{|c|c|c|c|}
\hline Régime & Manioc & Banane & $\begin{array}{l}\text { Significations } \\
\text { des différences }\end{array}$ \\
\hline \multicolumn{4}{|l|}{ Digestibilité } \\
\hline Matière sèche & $88,6 \pm 0,7$ & $87,4 \pm 0,9$ & $\mathrm{P}<0,01$ \\
\hline Matière organique & $90,5 \pm 0,6$ & $89,0 \pm 1,06$ & $P<0,01$ \\
\hline Azote .......... & $82,9 \pm 1,6$ & $80,9 \pm 1,7$ & $P<0,05$ \\
\hline Cendres......... & $63,4 \pm 2,3$ & $64,8 \pm 1,4$ & NS \\
\hline
\end{tabular}

Les coefficients d'utilisation digestive apparente de la matière sèche, de la matière organique et des protéines du régime à base de manioc sont supérieurs à ceux $\mathrm{du}$ régime à base de banane. Les différences sont significatives $(\mathrm{P}<0, \mathrm{OI})$ pour la matière sèche et la matière organique et à $\mathrm{P}<0,05$ pour les protéines. En ce qui concerne 1'amidon, sa digestibilité finale est très élevée car la teneur en amidon est à peine décelable dans les fèces et les valeurs (inférieures à I p. Ioo) sont dépourvues de signification pour le calcul de la digestibilité.

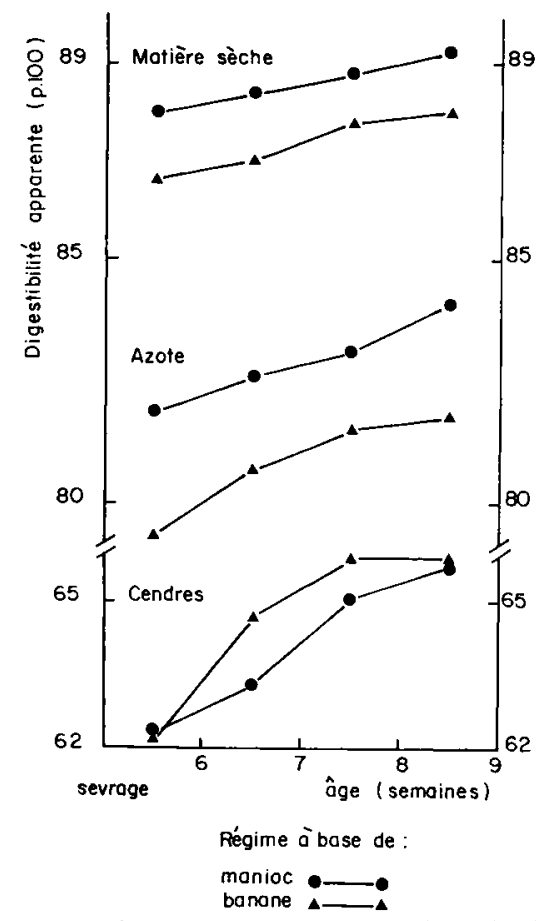

Hr.. I. - Évolution selon l'âge des porcelets, des coefficients de digestibilité apparente, de quelques éléments de la ration 
Par ailleurs, on constate une augmentation de tous les coefficients d'utilisation digestive avec l'âge des animaux (fig. I). Elle est particulièrement importante pour les protéines et les cendres, soit respectivement 2,2 et 3,4 points pour les régimes à base de manioc et 2,5 et 3,5 points pour les régimes à base de banane.

\section{DISCUSSION}

Les résultats d'ensemble font apparaître que la farine de banane possède pour le porcelet âgé de 5 à 9 semaines une valeur alimentaire comparable à celle de la farine de manioc. Il convient en particulier de souligner que contrairement à ce qui est observé avec le fruit vert entier chez le porc de $30 \mathrm{~kg}$ à l'abattage (LE DIvIDICH et Canope, I969; Seve, Le Dividich et Canope, I972), la banane deshydratée ne semble pas provoquer d'effet dépressif sur la croissance des animaux. Il apparaît d'ailleurs de plus en plus certain que c'est la forte teneur en eau (8o p. Ioo) qui constitue le facteur limitant essentiel de 1'utilisation de la banane par le Porc.

Dans nos conditions expérimentales, un taux de ig p. Ioo de protéines dans la ration est insuffisant pour permettre une croissance maximale. Nous pouvons par contre souligner que le taux de 2 I,5 p. Ioo, proche de l'optimum trouvé en milieu tempéré par SMith et LuCas, i957 ; Aumaitre, Jouandet et Salmon-L,EgaGNEUR, I964; MEADF et al., I969, et en milieu tropical par BABA'TUNDE et al., I97I, peut être recommandé lors de l'emploi des produits amylacés tropicaux dans une ration de sevrage à 5 semaines. Dans d'autres conditions (régimes à base de maïs et de tourteau de soja), WYLLIE et al., I969, obtiennent le maximum de croissance pour un taux de protéines inférieur ( $\mathrm{I}$ 7 p. Ioo). Cependant il ne nous semble pas possible de retenir de telles recommandations pour des animaux de race Large White acclimatés au milieu tropical. L'augmentation de l'efficacité alimentaire avec le taux de protéines est conforme aux résultats de MEADE et al., I969; WyLLIE et al., I969; BABATUNDE et al., I97I. En revanche, si l'on considère l'efficacité protidique, sa diminution, observée lorsque le taux d'azote augmente, a été maintes fois soulignée.

La digestibilité des divers éléments du régime à base de farine de banane, notamment la matière sèche et la matière organique, reste toujours inférieure (sauf pour les cendres) à celle du régime à base de farine de manioc. Néanmoins nos résultats montrent une digestibilité de la matière organique de la banane séchée plus élevée dans la présente expérience, que pour le fruit vert distribué au porc en croissance (Le, Dividich et Canope, I97i ; Seve, LE Dividich et CANope, 1972.) Cette amélioration peut être attribuée à une meilleure dégradation de l'amidon sous l'action conjuguée du séchage de la banane à forte température qui multiplie par 20 la quantité d'amidon facilement dégradable par rapport au produit frais (LE DIvidich et Cerning, I972) et de la granulation de l'aliment (MERCier et Guilbot, I962).

De plus, quel que soit le type de ration, la digestibilité de ses éléments augmente avec 1'âge des animaux en accord avec Llovo et al. (I957) ; AUMaITRE (I969); BAYLEY et CARLSON (I970), traduisant une adaptation digestive du jeune animal au régime de sevrage ainsi que l'ont montré DoLLAR et al. (1957). Soulignons enfin l'excellent état sanitaire des animaux qui constitue un facteur important dans le 
choix des aliments de sevrage. Dans nos conditions expérimentales, le jeune porcelet " tolère » la farine de pulpe de banane séchée aussi bien que les racines entières de manioc séchées.

En conclusion, la farine de banane séchée peut constituer un bon aliment de base pour le sevrage précoce du porcelet. Ellle peut être incorporée sans risque jusqu'à 50 p. roo dans le régime. Par ailleurs, de bonnes performances de croissance sont obtenues lorsque le taux de protéines dans la ration est voisin de 2I,5 p. Ioo.

Reçu pour publication en décembre 1973.

\section{SUMMARY}

FEEDING VALUE OF BANANA MEAL, AND MANIOC MEAI, IN DIETS OF PIGLETS WEANED AT 5 WEEKS : EFFECT OF THE PROTEIN LEVEL, OF THE, RATION

Two experiments were conducted on 62 piglets weaned at five weeks in order to compare the feeding value of manioc meal with banana meal when incorporated at about $52 \mathrm{p}$. Ioo into the diet.

Two levels of protein (19.0 and $2 \mathrm{I} .5 \mathrm{p}$. IOo) were taken into consideration. The diets were pelleted and the animals fed ad libitum.

There was no significant difference between the performance of the animals fed the diets based either on manioc meal or banana meal. On the other hand, the high level of protein (21.5 p. IOO) improved significantly the average daily gain $(\mathrm{P}<0.0 \mathrm{I})$ and feed efficiency $(\mathrm{P}<0.05)$.

The apparent digestibility of the diet based on manioc meal was significantly higher for dry matter and organic matter $(\mathrm{P}<0.0 \mathrm{I})$ and protein $(\mathrm{P}<0.05)$.

The frequency of diarrhoea was low and the use of these tropical products may be recommended up to $52 \mathrm{p}$. Ioo of the diet with a protein level of about $21.5 \mathrm{p}$. Ioo for the weaning of 5 weeks old piglets.

\section{RÉFÉRENCES BIBLIOGRAPHIQUES}

Aumaitre A., I969. Valeur alimentaire du manioc et de différentes céréales dans les régimes de sevrage précoce du Porcelet : utilisation digestive de l'aliment et effet sur la croissance des animaux. Ann. Zootech. 18, 385-398.

Aumaitre A., Jouandet C., Salmon-Legagneur E., I964. Effet des taux énergétique et protidique de la ration sur l'efficacité alimentaire et sur la croissance chez le Porcelet. Ann. Zootech. 13, $24 \mathrm{r}-253$.

Aumaitre A., Corring T., Le Dividich J., I969. Étude de la vitesse d'hydrolyse in vitro de quelques amidons de plantes tropicales (Patate douce, Banane, Igname) par le suc pancréatique de Porcelet; relation entre la vitesse de dégradation in vitro et la digestibilité apparente de la ration, Journées Rech. Porcine en France, 99-103, I. N. R. A., Paris. I. 'T. P. éd.

Babatunde G. M., Olomu J., Oyenuga V. A., I97I. Determination of the optimum crude protein requirement of pigs in a tropical environment. Anim. Prod. 14, 57-67.

Bailey C. B., Kitss W. D., Woods A. J. x956. The development of the digestive enzyme system of the pig during its preweaning phase of growth. B. intestinal lactose, sucrose and maltose. Canad. J. A gric. Sci. 36, $5 \mathrm{I}-58$.

Bayley H. S., Carlson W. E., ig7o. Comparison of simple and complex diets for baby pigs : effect of form of feed and of glucose addition. J. Anim. Sci. 30, 394-4or.

Bolin D. W., King R. P., Klosterman E. W., 1952, A simplified method for the determination of chromic oxide when used as an index substance. Science, 116,634-635.

Cunningham H. M., I959. Digestion of starch and some of its degradation products by new-born pigs. J. Anim. Sci. 18, 964-974.

CunNingham H. M., Brisson G., 1957. The effect of amylases on the digestibility of starch by baby pigs. J. Anim. Sci. 16, 370-375. 
Dollar A. M., Mitchell K. G., Porter J. W. G., I957. The utilization of carbohydrates in the young pig. Proc. Nutr. Soc. 16, XII-XIII.

Le DIVIDICH J., CANOPE 1., I969. Utilisation des déchets de banane par le porc en croissance : premiers résultats. Journées Rech, Porcine en France. I3I-I35. I. N. R. A., Paris., I. T. P. ed.

Lloyd L. E., Crampton E. W., Mac Kay V. G., I957. The digestibility of ration nutrients by three VS seven weeks old pigs. J. Anim. Sci. 16, 383-387.

Meade R. J., Vermedahl D. L., Rust J. W., Wass D. F., ig69. Effect of protein content of the diet of the young pig on rate and efficiency of gain during early development and subsequent to $23,5 \mathrm{~kg}$ and carcass characteristics and composition of lean tissue. J. Anim. Sci. 28, 473-478.

Mercier Ch., Guilbot A., I962. Répercussions sur la digestibilité de l'amidon des modifications de sa structure physico-chimique au cours de ses transformations technologiques. Indust. alim. agric. 11, 939-947.

Oyenuga G. A., I96r. Nutritive value of cereal and cassava diets for growing and fattening pigs in Nigeria. Brit. J. Nutr. 15, 327-338.

Salmon-Legagneur E., FÉvrier R., i959. Les préférences alimentaires des porcelets. III. Appétence de quelques céréales. Ann. Zootech. 8, I39-I46.

Seve B., Le Dividich J., Canope I., ig72. Utilisation des déchets de banane par le porc aux Antilles Françaises. Essais de conservation par ensilage. Journées Rech. Porcine en France. Paris, 207-213, I. T. P. ed.

Smith H., Lucas I. A. M., r957. The early weaning of pigs. III. The influence of protein supply during two stages of growth on the performance of pigs from $\mathrm{g}$ lb to bacon weight. J. A gric. Sci. 49, 409-4I7.

Terroine E. F., r93r. De l'emplei des laits artificiels dans l'élevage du bétail. Bull. Soc. Sci. Hyg. Aliment. Paris 19, I-23.

Thivend P., Mercier Ch., Guilbot A., I965. Dosage de l'amidon dans les milieux complexes. Ann. Biol. anim. Bioch. Biophys, 5, 513-526.

Walker D. M., I959. The development of the digestive system of the young animal. II. Carbohydrase enzyme development in the young pig. J. A gric. Sci. 52, 357-37o.

WeIJers H. A., VAN DE KAMER, J. H. I965. Alteration of intestinal bacterial flora as a cause of diarrhoea. Nutr. Abstr. Rev. 35, 59I-604.

Wyllie D., Speer V. C., Ewan R. C., Hays V. W., I969. Effects of starter protein level on performance and body composition of pigs. J. Anim. Sci. 29, 433-438. 